\title{
Laparoscopic Submucosal Dissection for Gastrointestinal Stromal Tumor of the Stomach: A Novel Technique for Local Excision with a Minimal Curative Margin
}

\author{
Norihito Wada ${ }^{1 *}$, Yoshiro Saikawa ${ }^{1}$, Hiroya Takeuchi ${ }^{1}$, Tsunehiro Takahashi ${ }^{1}$, Rieko Nakamura ${ }^{1}$, \\ Hirofumi Kawakubo ${ }^{1}$, Kaori Kameyama ${ }^{2}$, Makio Mukai ${ }^{2}$, Yuko Kitagawa ${ }^{1}$ \\ ${ }^{1}$ Department of Surgery, School of Medicine, Keio University, Tokyo, Japan \\ ${ }^{2}$ Departments of Pathology, School of Medicine, Keio University, Tokyo, Japan \\ Email: *nori-kkr@umin.ac.jp
}

Received June 16, 2012; revised July 28, 2012; accepted August 9, 2012

\begin{abstract}
Background: Laparoscopic wedge resection is accepted as a curative treatment for small- and medium-sized gastrointestinal stromal tumors (GISTs) of the stomach. Conventional methods involving surgical staplers require relatively large lateral margins, which may cause deformity and postoperative dysfunction of the gastric remnant. In this study, we introduce a novel technique called laparoscopic submucosal dissection (LSD) in which the defects of the stomach are minimized and a microscopic negative margin is secured. Methods: The normal seromuscular layer of the gastric wall was dissected with a $5 \mathrm{~mm}$ lateral margin. Then, the submucosal tissue was divided carefully using a monopolar electrosurgical device with a curved spatula tip. Results: The operation time was $170 \mathrm{~min}$, and the amount of bleeding was very small. We confirmed an intact pseudo-capsule and marginal subserosal or submucosal tissue of the tumor by histological analysis. The postoperative course was uneventful with no complications. Endoscopy showed minimal deformity of the gastric remnant. Conclusion: We think that LSD is a curative and less invasive treatment for GIST of the stomach. Further investigations are necessary to evaluate the oncological and functional outcomes of this procedure.
\end{abstract}

Keywords: Gastrointestinal Stromal Tumor; Submucosal Tumor; Endoscopic Submucosal Dissection; Wedge Resection; Laparoscopic Surgery; Minimally Invasive Surgery

\section{Introduction}

Gastrointestinal stromal tumors (GISTs) represent only $0.1 \%$ to $3 \%$ of all gastrointestinal malignancies, but they are the most common (approximately 80\%) mesenchymal tumors of the gastrointestinal tract [1]. The estimated incidence of GIST is 10 - 20 per million people per year. These tumors can arise from throughout the gastrointestinal tract, but $60 \%$ arise from the stomach [2]. Relatively small GISTs are usually asymptomatic and diagnosed incidenttally as submucosal tumor (SMT) of the stomach. Although the management of gastric SMTs used to be controversial, Japanese guidelines for GIST [3] recommended that histologically established GIST is an indication for surgery regardless of the size of the tumor. Furthermore, laparoscopic surgery can be considered if the tumor is $5 \mathrm{~cm}$ or less. Principles of GIST surgery include complete surgical resection, no injury to the pseudo-capsule, a macroscopically negative margin with an adequate safety margin, and partial resection without prophylactic lymph node dissection [3-5]. When these criteria are met, laparoscopic surgery is

${ }^{*}$ Corresponding author. indicated for patients with small- or medium-sized GISTs of the stomach.

In laparoscopic wedge resection, stapled sutures are preferred in most cases [6] because this is a simple and easy method. However, excessive amounts of the normal gastric wall may be resected along with the tumor, resulting in deformity of the gastric remnant and sometimes malfunction of the stomach. In this study, we present a novel technique called laparoscopic submucosal dissection (LSD), in which defects of the stomach are minimized and the curative margin is secured.

\section{Materials and Methods}

\subsection{Patient}

A 52-year-old man with an SMT (26 mm in diameter) in the middle body of the stomach visited our hospital. This patient was asymptomatic and was otherwise healthy. A gastric SMT was diagnosed 5 years ago. The diameter of the tumor had increased from 16 to $26 \mathrm{~mm}$. An abdominal computed tomography (CT) scan (Figure 1(a)), $\mathrm{X}$-ray (Figure 1(b)), endoscopy (Figure 1(c)), and endoscopic ultrasonography (Figure 1(d)) revealed typical 


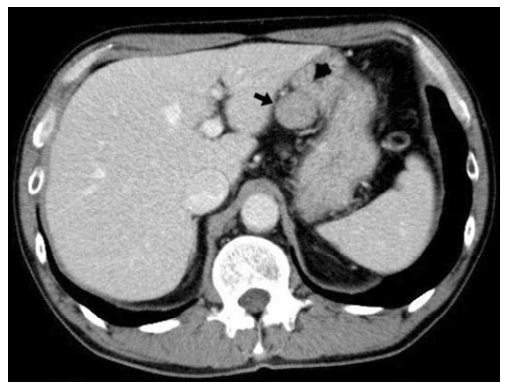

(a)

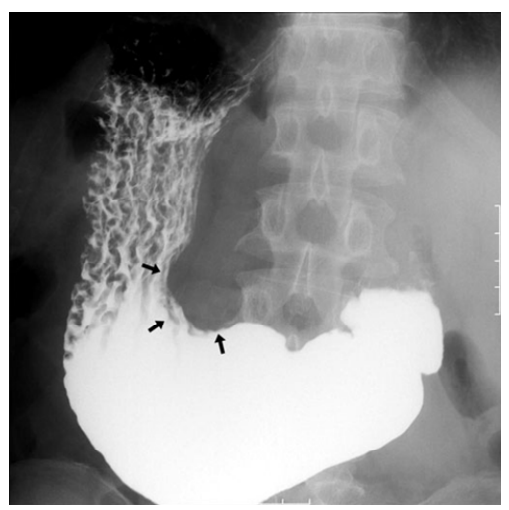

(b)

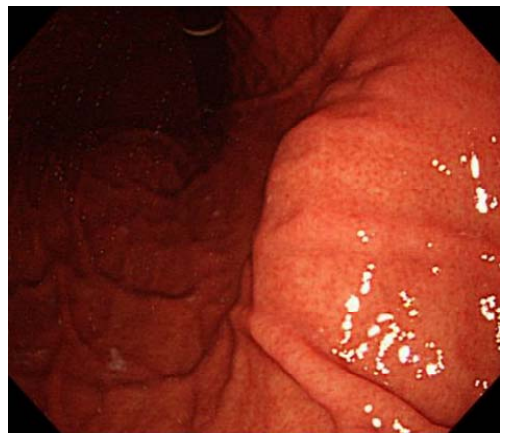

(c)

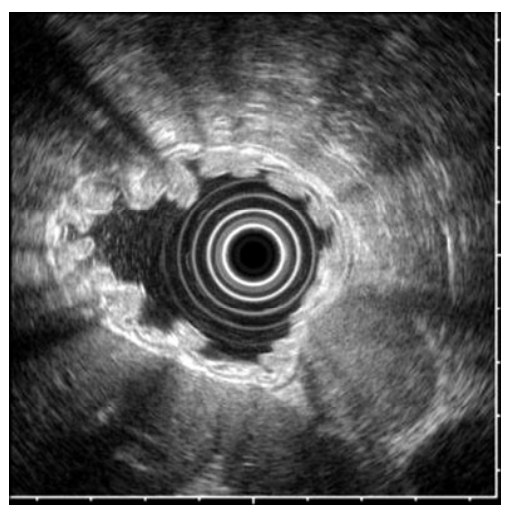

(d)

Figure 1. Preoperative evaluation of the tumor. Computed tomography (CT), X-ray, endoscopy, and endoscopic ultrasonography revealed typical findings of a gastric mesenchymal tumor located in the lesser curvature of the gastric body. (a) Enhanced CT scan; (b) Barium meal X-ray; (c) Endoscopy; (d) Endoscopic ultrasonography. findings of gastric mesenchymal tumors located in the middle part of the lesser curvature. Written informed consent was obtained for this surgery.

\subsection{Surgical Procedures}

All the surgical procedures were performed laparoscopically with the patient under general anesthesia and in the supine position. The split leg position was used to allow the laparoscopist to stand between the patient's legs. The primary surgeon stood on the patient's right with the first assistant on the left. Five laparoscopic ports were created in the patient's upper abdomen. Pneumoperitoneum with carbon dioxide under a pressure of 8 - $10 \mathrm{~mm} \mathrm{Hg}$ was created. A high-definition 10-mm flexible laparoscope was inserted through the 12-mm port in the inferior umbilical area.

To confirm the location of the tumor, the serosal side of the gastric wall was carefully observed (Figure 2(a)). The tumor was found to be located in the middle part of the lesser curvature and was covered with an intact serosa. The lesser omentum was dissected with ultrasonic coagulation scissors. The seromuscular layer of the normal gastric wall adjacent to the tumor was dissected by use of a monopolar electrosurgical device (ForceTriVerse ${ }^{\circledR}$ in conjunction with Opti2 ${ }^{\circledR}$ electrodes, Covidien, MA) with an energy platform (ForceTriad ${ }^{\circledR}$, Covidien, MA). The normal muscular layer surrounding the tumor is important for handling the tumor because direct grasping of the tumor might cause it to rupture. We secured a 5-mm lateral margin around the tumor. After the submucosal and proper mucosal layers underneath were observed, we carefully used the LSD method with a monopolar electrosurgical device. Blunt dissection is a safe and reliable technique to prevent injury to the tumor or proper mucosal layer. Moreover, a curved spatula tip is preferable because small vessels are easily coagulated and dissected bluntly with this type of an electrode tip (Figure 2(b)). After the resection was completed, the tumor was immediately collected in a bag. Intact proper mucosal layer was observed at the bottom of the gastric wall defect (Figure 2(c)).

All the laparoscopic procedures were carefully performed without any damage to the pseudo-capsule of the GIST. Throughout the dissection, the active blade of the ultrasonic coagulation device was placed on the opposite side of the tumor. The seromuscular layer was laparoscopically approximated with monofilament absorbable strings (Figure 2(d)). Intraoperative endoscopy was effectively used to confirm that the mucosa was unbroken and that the stomach was airtight after serosal closure (Figure 2(e)).

\section{Results}

This procedure was performed safely without any intraoperative complications. The operation time was $170 \mathrm{~min}$, 


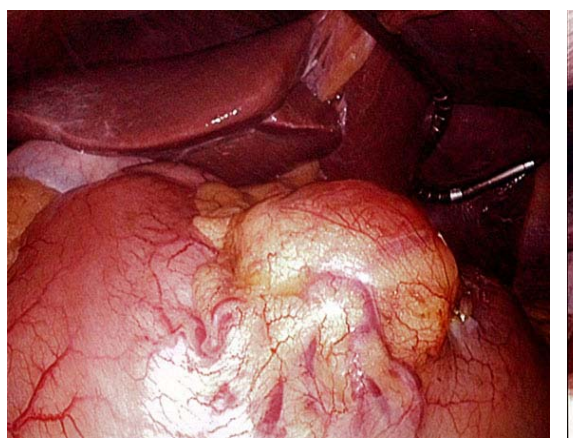

(a)

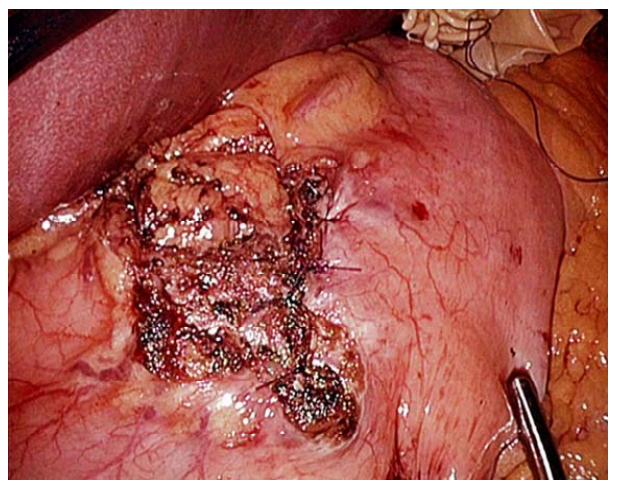

(d)

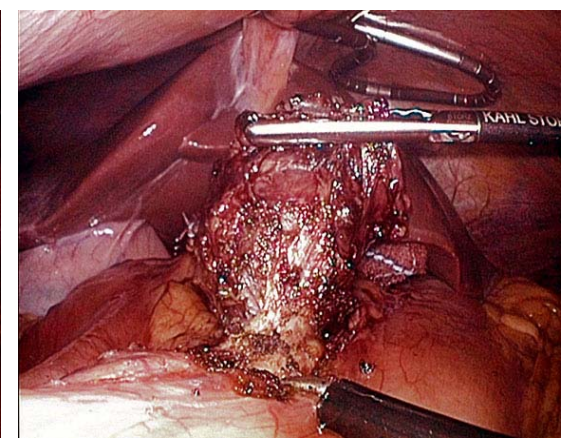

(b)

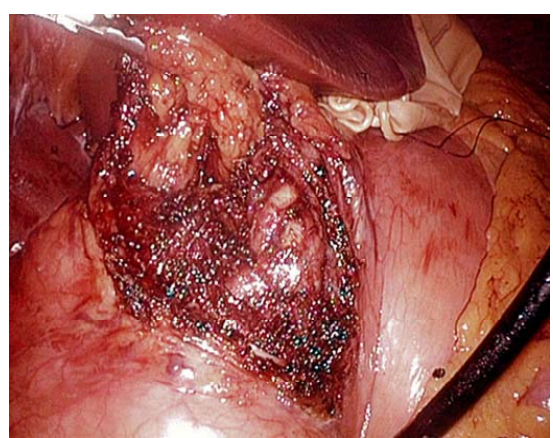

(c)

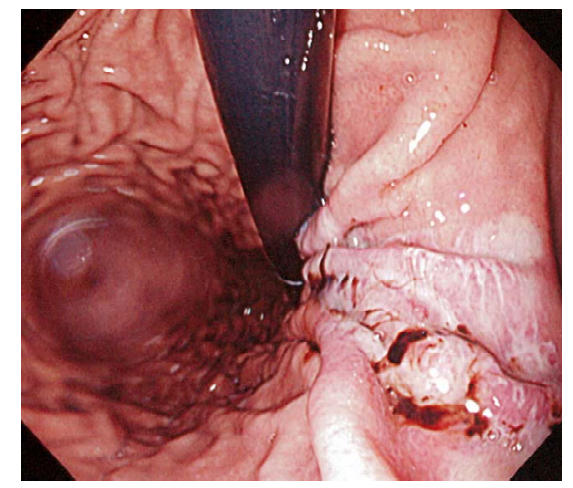

(e)

Figure 2. (a) Laparoscopic view of the gastric gastrointestinal stromal tumor (GIST). The tumor was located in the middle part of the lesser curvature and was covered with an intact serosa; (b) Laparoscopic submucosal dissection (LSD). The tumor had a sufficient lateral margin of normal gastric wall around it and was handled with an atraumatic-hollow jaw grasper, which does not grasp the tumor itself but grasps the brim of the tumor. The tumor had an intact pseudo-capsule and was covered with submucosal tissue on the mucosal surface. A curved spatula tip is preferable for blunt dissection during LSD; (c) Laparoscopic view of the gastric wall after the tumor was removed. The tumor was collected in a bag. Intact proper mucosal layer was observed at the bottom of the gastric wall defect; (d) Laparoscopic view after the seromuscular layer was approximated with monofilament absorbable strings; (e) Endoscopic view after seromuscular closure. Intraoperative endoscopy confirmed the closed mucosa after LSD and air tightness of the stomach.

and the amount of bleeding was very small. The size of the tumor was $30 \times 28 \times 25 \mathrm{~mm}$ with an exophytic growth pattern. The tumor was covered with an intact pseudocapsule. Immunohistochemical analysis showed that it was positive for KIT and CD34 but negative for SMA, desmin, and S-100, which indicates that the tumor was a GIST. Mitosis was observed 3/50HPF with $2 \%-3 \%$ MIB-1-positive cells. Histological analysis showed that the tumor was covered with an intact pseudo-capsule and subserosal tissue (Figure 3(a)), and the presence of marginal submucosal tissue on the pseudo-capsule was confirmed (Figure 3(b)). A mutation in the c-kit gene was detected in the coding region of exon 11.

The postoperative course was uneventful with no complications. The patient's dietary intake and body weight remained unchanged after surgery. No adjuvant therapy was administered. We followed the patient for 21 months with periodic CT scanning and upper gastrointestinal endoscopy, but we did find any signs of recurrence or postoperative complaints. Deformity of the area of excision was minimal, and stenotic changes were not observed (Figure 4).

\section{Discussion}

We have reported the operative indications for relatively small GISTs of the stomach [7]. Surgical resection is the gold standard for treatment of nonmetastatic primary GISTs as indicated in published guidelines [3,4]. The principles of GIST surgery include no injury to the pseudo-capsule and macroscopically negative margins [3]. In this context, the laparoscopic approach to surgical treatment of gastric GIST is a safe and effective option for patients with small- and medium-sized tumors if treated appropriately [8-10]. In this study, we secured a 5 mm lateral margin around the tumor, and normal muscular tissue was used to handle the tumor to avoid injury to the pseudo-capsule of the GIST. Furthermore, we carefully dissected the submucosal tissue under the magnified view afforded by the laparoscope, and the presence of marginal submucosal tissue was confirmed by histological analysis. This dissection method would be difficult for use 


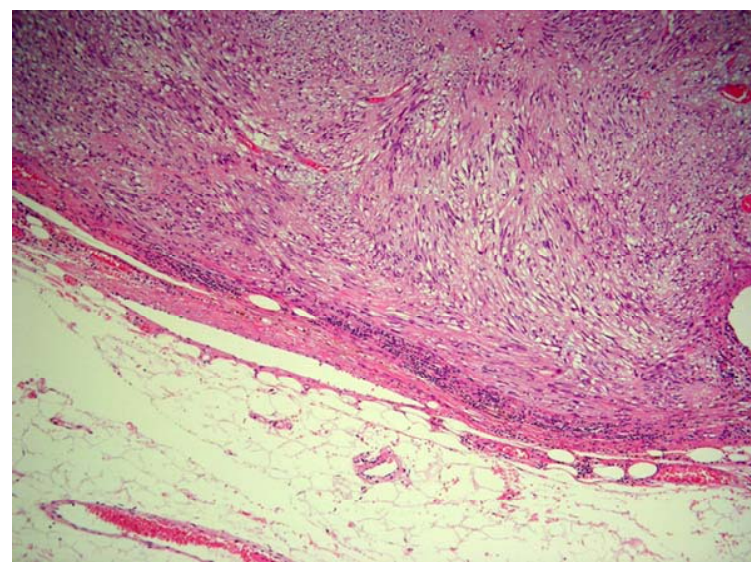

(a)

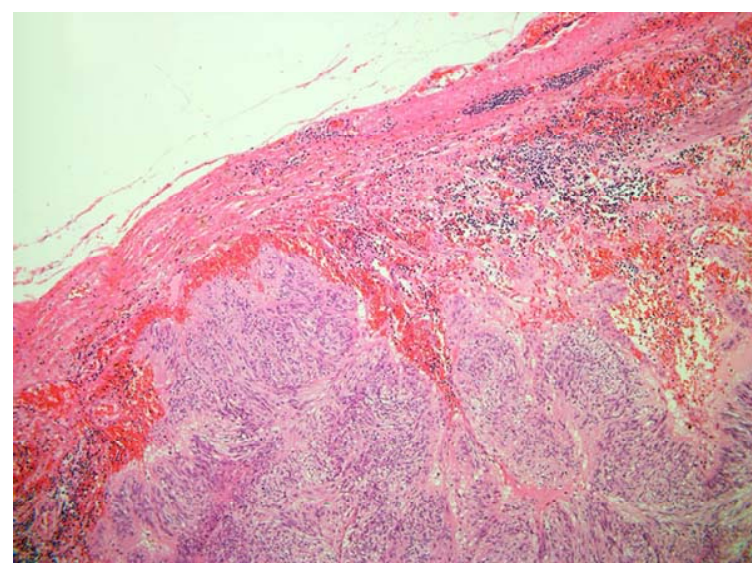

(b)

Figure 3. Histology of the resected specimen (hematoxylin and eosin stain). (a) Serosal side of the tumor (original magnification $\times 40$ ). The tumor was covered with an intact pseudo-capsule and subserosal tissue; (b) Mucosal side of the tumor (original magnification $\times 200$ ). Intact pseudocapsule and marginal submucosal tissue were confirmed by histological analysis.

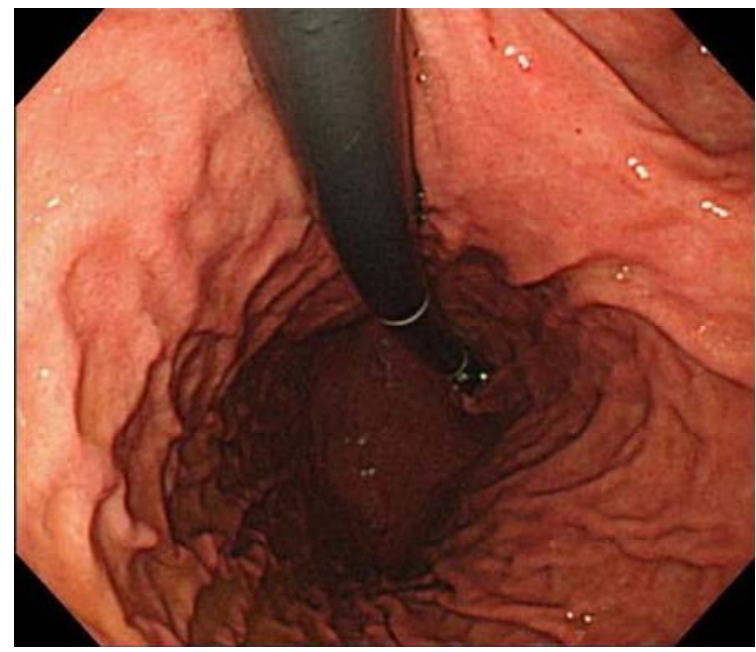

Figure 4. Postoperative endoscopy after 15 months. Stenosis and significant deformity were not observed. in open surgery because the resolution of the human eye would not be sufficient to distinguish the adequate layer for dissection. Laparoscopic surgery enables us to divide the submucosal tissue precisely and to find a microscopically negative margin. In this aspect, endoscopic submucosal dissection (ESD) is comparable with LSD. The ESD method has been established as a promising endoscopic therapy for early gastric cancer [11]. The vertical margin of the tumor is clearly visualized in ESD. In LSD, the submucosal layer can also be seen as clearly, which enables us to create a secure margin during submucosal dissection.

Katoh and coworkers reported the endoscopic enucleation of gastric GISTs [12]. The indication of endoscopic enucleation is limited to tumors arising from the submucosa leaving the muscularis propria free of the tumor. Furthermore, the size of the tumor has to be less than 3 $\mathrm{cm}$ because larger tumors cannot be retrieved from the mouth. In this report, the authors showed that tumor resection with submucosal dissection can be used to achieve complete resection of the tumor and for a curative oncologic outcome.

The characteristics of LSD include 1) minimal deformity of the stomach, 2) clean surgery without stomach exploration, and 3) lower costs and smaller ports because of the lack of stapling devices. However, this procedure has disadvantages such as 1) unknown oncological validity, 2) technically advanced surgery, and 3) a relatively longer operation time. From our limited experience, however, these drawbacks of LSD are limited, and this new technique is feasible.

Hiki et al. [13] reported the usefulness of laparoscopic and endoscopic cooperative surgery (LECS) for gastric wedge resection of gastric SMT. In comparison with LECS, LSD can lead to less deformity and create a cleaner environment during surgery. They have also reported that the success of the procedure does not depend on tumor location, such as the vicinity of the esophagogastric junction or pyloric ring. We performed LSD for a patient with benign SMT of the cardia, and the results of the surgery were as good as those for the method pre- sented here (data not shown).

Natural orifice translumenal endoscopic surgery (NOTES) [14] may be a less invasive treatment for gastric GIST. This type of partial gastrectomy would minimize the size of the abdominal wound and the amount of postoperative pain. However, the invasiveness of the NOTES procedure in the stomach are the same as that for laparoscopic surgery. Therefore, LSD would have better outcomes in terms of surgical site infections and postoperative stomach function.

In LSD, surgical staplers are not required, which means that the cost and size of the trocars are reduced compared with conventional laparoscopic surgery. 


\section{Conclusion}

We suggest that LSD is a curative and less invasive treatment for GIST of the stomach. Further investigations are necessary to evaluate the oncological and functional outcomes of this novel procedure.

\section{Disclosure}

Drs. N. Wada, Y. Saikawa, H. Takeuchi, T. Igarashi, T. Oyama, T. Takahashi, R. Nakamura, K. Kameyama, M Mukai, Y. Kitagawa have no conflicts of interest or financial ties to disclose.

\section{REFERENCES}

[1] M. Scarpa, M. Bertin, C. Ruffolo, L. Polese, D. F. D’Amico and I. Angriman, "A Systematic Review on the Clinical Diagnosis of Gastrointestinal Stromal Tumors,” Journal of Surgical Oncology, Vol. 98, No. 5, 2008, pp. 384-392. doi:10.1002/jso. 21120

[2] P. Gupta, M. Tewari and H. S. Shukla, "Gastrointestinal Stromal Tumor,” Surgical Oncology, Vol. 17, No. 2, 2008, pp. 129-138. doi:10.1016/j.suronc.2007.12.002

[3] T. Nishida, S. Hirota, A. Yanagisawa, Y. Sugino, M. Minami, Y. Yamamura, Y. Otani, Y. Shimada, F. Takahashi and T. Kubota, "Clinical Practice Guidelines for Gastrointestinal Stromal Tumor (GIST) in Japan: English Version," International Journal of Clinical Oncology, Vol. 13, No. 5, 2008, pp. 416-430. doi:10.1007/s10147-008-0798-7

[4] G. D. Demetri, R. S. Benjamin, C. D. Blanke, J. Y. Blay, P. Casali, H. Choi, C. L. Corless, M. Debiec-Rychter, R. P. DeMatteo, D. S. Ettinger, G. A. Fisher, C. D. Fletcher, A. Gronchi, P. Hohenberger, M. Hughes, H. Joensuu, I. Judson, A. Le Cesne, R. G. Maki, M. Morse, A. S. Pappo, P. W. Pisters, C. P. Raut, P. Reichardt, D. S. Tyler, A. D. Van den Abbeele, M. von Mehren, J. D. Wayne and J. Zalcberg, "NCCN Task Force Report: Management of Patients with Gastrointestinal Stromal Tumor (GIST) -Update of the NCCN Clinical Practice Guidelines," Journal of the National Comprehensive Cancer Network, Vol. 5, Suppl. 2, 2007, pp. S1-S29, Quiz: S30.

[5] J. Y. Blay, S. Bonvalot, P. Casali, H. Choi, M. Debiec-Richter, A. P. Dei Tos, J. F. Emile, A. Gronchi, P. C. Hogendoorn, H. Joensuu, A. Le Cesne, J. McClure, J. Maurel, N. Nupponen, I. Ray-Coquard, P. Reichardt, R. Sciot, S. Stroobants, M. van Glabbeke, A. van Oosterom and G. D. Demetri, "Consensus Meeting for the Management of Gastrointestinal Stromal Tumors: Report of the GIST Consensus Conference of 20-21 March 2004, under the Auspices of ESMO," Annals of Oncology, Vol. 16, No. 4, 2005, pp. 566-578. doi:10.1093/annonc/mdi127

[6] F. Catena, M. Di Battista, P. Fusaroli, L. Ansaloni, V. Di
Scioscio, D. Santini, M. Pantaleo, G. Biasco, G. Caletti and A. Pinna, "Laparoscopic Treatment of Gastric Gist: Report of 21 Cases and Literature's Review,” Journal of Gastrointestinal Surgery, Vol. 12, No. 3, 2008, pp. 561568. doi:10.1007/s11605-007-0416-4

[7] Y. Otani, T. Furukawa, M. Yoshida, Y. Saikawa, N. Wada, M. Ueda, T. Kubota, M. Mukai, K. Kameyama, Y. Sugino, K. Kumai and M. Kitajima, "Operative Indications for Relatively Small (2 - $5 \mathrm{~cm}$ ) Gastrointestinal Stromal Tumor of the Stomach Based on Analysis of 60 Operated Cases,” Surgery, Vol. 139, No. 4, 2006, pp. 484492. doi:10.1016/j.surg.2005.08.011

[8] Y. W. Novitsky, K. W. Kercher, R. F. Sing and B. T. Heniford, "Long-Term Outcomes of Laparoscopic Resection of Gastric Gastrointestinal Stromal Tumors," Annals of Surgery, Vol. 243, No. 6, 2006, pp. 738-747. doi:10.1097/01.sla.0000219739.11758.27

[9] G. R. Silberhumer, M. Hufschmid, F. Wrba, G. Gyoeri, S. Schoppmann, B. Tribl, E. Wenzl, G. Prager, F. Laengle and J. Zacherl, "Surgery for Gastrointestinal Stromal Tumors of the Stomach,” Journal of Gastrointestinal Surgery, Vol. 13, No. 7, 2009, pp. 1213-1219. doi:10.1007/s11605-009-0872-0

[10] S. M. Choi, M. C. Kim, G. J. Jung, H. H. Kim, H. C. Kwon, S. R. Choi, J. S. Jang and J. S. Jeong, "Laparoscopic Wedge Resection for Gastric GIST: Long-Term Follow-Up Results,” European Journal of Surgical Oncology, Vol. 33, No. 4, 2007, pp. 444-447. doi:10.1016/j.ejso.2006.11.003

[11] I. Oda, T. Gotoda, H. Hamanaka, T. Eguchi, Y. Saito, T. Matsuda, P. Bhandari, F. Emura, D. Saito and H. Ono, "Endoscopic Submucosal Dissection for Early Gastric Cancer: Technical Feasibility, Operation Time and Complications from a Large Consecutive Series,” Digestive Endoscopy, Vol. 17, No. 1, 2005, pp. 54-58. doi:10.1111/j.1443-1661.2005.00459.x

[12] T. Katoh, Y. Itoh, T. Mohri and H. Suzuki, "Endoscopic Enucleation of Gastrointestinal Stromal Tumors of the Stomach: Report of Five Cases,” World Journal of Gastroenterology, Vol. 14, No. 16, 2008, pp. 2609-2611. doi:10.3748/wjg.14.2609

[13] N. Hiki, Y. Yamamoto, T. Fukunaga, T. Yamaguchi, S. Nunobe, M. Tokunaga, A. Miki, S. Ohyama and Y. Seto, "Laparoscopic and Endoscopic Cooperative Surgery for Gastrointestinal Stromal Tumor Dissection,” Surgical Endoscopy, Vol. 22, No. 7, 2008, pp. 1729-1735. doi:10.1007/s00464-007-9696-8

[14] K. Nakajima, T. Nishida, T. Takahashi, Y. Souma, J. Hara, T. Yamada, T.Yoshio, T. Tsutsui, T. Yokoi, M. Mori and Y. Doki, "Partial Gastrectomy Using Natural Orifice Translumenal Endoscopic Surgery (NOTES) for Gastric Submucosal Tumors: Early Experience in Humans,” Surgical Endoscopy, Vol. 23, No. 12, 2009, pp. 2650-2655. doi:10.1007/s00464-009-0474-7 